\title{
USING X-RAYS TO PROBE THE COMPACT BINARY CONTENT OF GLOBULAR CLUSTERS
}

\author{
N. A. Webb, ${ }^{1}$ B. Gendre, ${ }^{1}$ and D. Barret ${ }^{1}$
}

\begin{abstract}
RESUMEN
Los cúmulos globulares (GCs) albergan un gran número de binarias cerradas que son difíciles de identificar ópticamente debido a las altas densidades estelares. Observando estos GCs en rayos X donde las binarias compactas son brillantes podemos disminuir este problema. Utilizando los observatorios de rayos $\mathrm{X}$ de nueva generación es posible identificar poblaciones de binarias de rayos $\mathrm{X}$ de estrellas de neutrones de baja masa, variables cataclísmicas, pulsares de milisegundos y también otros tipos de binarias. Presentamos los espectros de una variedad de binarias que hemos identificado en cuatro GCs observados por XMM-Newton. Mostramos que basándonos en estudios de poblaciones podemos empezar a entender la formación de las clases individuales de binarias en GCs y comenzar a revelar los caminos evolutivos de estos sistemas.
\end{abstract}

\section{ABSTRACT}

Globular clusters (GCs) harbour a large number of close binaries which are hard to identify optically due to high stellar densities. Observing these GCs in X-rays, in which the compact binaries are bright, diminishes the over-crowding problem. Using the new generation of X-ray observatories, it is possible to identify populations of neutron star low mass X-ray binaries, cataclysmic variables and millisecond pulsars as well as other types of binaries. We present the spectra of a variety of binaries that we have identified in four GCs observed by XMMNewton. We show that through population studies we can begin to understand the formation of individual classes of binaries in GCs and hence start to unfold the complex evolutionary patins of these systems.

Key Words: GLOBULAR CLUSTERS: GENERAL - STARS: NEUTRON - X-RAYS: BINARIES

\section{GENERAL}

It is expected that GCs should contain many binary systems, due to interactions occurring within the clusters (e.g. Di Stefano \& Rappaport 1994). These systems could play a critical role in the dynamical evolution of GCs, serving as an internal energy source which counters the tendency of cluster cores to collapse (see Hut et al. 1992 for a review). The binaries are difficult to locate, because of high stellar densities. However, the binaries are also visible in X-rays, where the crowding is less severe. Indeed the small population of bright $\mathrm{X}$-ray sources $\left(\mathrm{L}_{x}>10^{36} \mathrm{erg} \mathrm{s}^{-1}\right)$, known to be $\mathrm{X}$-ray binaries (Hertz \& Grindlay 1983), were detected primarily through their X-ray bursts. However, there is also a population of low-luminosity $\left(\mathrm{L}_{x} \lesssim 10^{34.5} \mathrm{erg} \mathrm{s}^{-1}\right)$ $\mathrm{X}$-ray sources. The membership of this population has rapidly increased thanks to the new X-ray observatories; XMM-Newton and Chandra. A variety of objects have been identified, e.g: quiescent neutron star low mass X-ray binaries (qNSs) (e.g. Gendre, Barret, \& Webb 2003b; Rutledge et al. 2003); cataclysmic variables (e.g. Carson, Cool, \& Grindlay 2000; Gendre, Barret, \& Webb 2003a); millisecond

\footnotetext{
${ }^{1}$ CESR, Toulouse, France.
}

pulsars (e.g. Grindlay et al. 2001); active binaries (e.g. Kaluzny et al. 1996); and fore- and background objects, i.e. stars (e.g. Gendre et al. 2003a) or clusters of galaxies (e.g. Webb et al. 2004).

Outside GCs, qNSs are found to have soft X-ray spectra and luminosities of $10^{32} \lesssim \mathrm{L}_{\mathrm{x}}<10^{33} \mathrm{erg} \mathrm{s}^{-1}$. During outbursts, reactions deep in the crust heat the neutron star (Brown et al. 1998). Between outbursts, the heated surface radiates a thermal spectrum, emitted by a neutron star hydrogen atmosphere (NSA). Thus the combination of a soft NSA X-ray spectrum and $10^{32} \underset{\sim}{\sim} \mathrm{L}_{\mathrm{x}} \sim 10^{33} \mathrm{erg} \mathrm{s}^{-1}$ has been used to detect qNSs in GCs. Theory predicts that the qNSs are formed through stellar encounters (tidal capture/ exchange encounters) in the cores of GCs (Verbunt 2002).

Taking advantage of XMM-Newton's sensitivity. we used spectral and timing studies to identify a rariety of low-luminosity X-ray sources in four GCs. Using these results and those from other X-ray observations, we study the formation of qNSs in GCs.

\section{DATA AND ANALYSIS}

We observed the four GC: M 22 (NGC 6656): $\omega$ Centauri (NGC 5139) M 13 (NGC 6205); and NGC 6366 with the EPIC cameras aboard IML- 
Newton. We used the full-frame imaging mode and the medium filter. After screening for high background activity, 20-40 ks of good observation time remained. The data have been reduced with V. 5.4.1 of the XMM-Newton SAS (Science Analysis Software). The MIOS data were reduced using 'emchain'. The event lists were filtered to retain only $0-12$ of the predefined patterns (single, double, triple, and quadruple pixel events). We used the energy range 0.2-10.0 $\mathrm{keV}$, as recommended in by Kirsch et al. (2002). The PN data were reduced using the 'epchain' of the SAS. Again the event lists were filtered, so that 0-4 of the predefined patterns (single and double events) were retained, as these have the best energy calibration and we used the energy range $0.5-10.0 \mathrm{kcV}$.

The source detection was done in the same way as Gendre et al. (2003a,b); Webb et al. (2004), but briefly we used the SAS wavelet detection algorithm on the $0.5-5.0 \mathrm{keV}$ images. We kept only those sources detected on at least two cameras, with the exception of PN only detections, as this camera is more sensitive than the MOS cameras. We detected $60,146,77,48$ sources respectivcly, with a maximum likelihood greater than 10 (correcting for the error in the SAS, V. 5.4.1). We estimated, using the Log NLog S relationship of extragalactic sources derived from the Lockman Hole (Hasinger et al. 2001), the background source population and thus the number of sources belonging to the clusters. In each case, the number of sources that belong to the GC is $5-10 \%$, with the exception of $\omega$ Centauri, where as many as $30 \%$ of the sources may belong to the cluster.

\section{RESULTS AND DISCUSSION}

Mass segregation should concentrate close binaries towards the core. Indeed we find this to be the case for M 22, M 13 and NGC 6366. However, $\omega$ Centauri is the exception, which may indicate that this cluster has been disrupted (see Gendre et al. 2003a). This cluster is also unusual as it contains a large population of active binaries. We have discovered a soft X-ray source in M 13, which is well fitted by a NSA model, with the radius and temperature expected from a neutron star (Gendre et al. 2003b). Its luminosity is also consistent with that of a qNS. There is a similar source in $\omega$ Centauri. A reasonable question to ask is whether we expect so many qNSs? Thus we studied all the XMM-Newton and Chan$d r a$ GC observations, where the luminosity limits are $\sim 10^{30}-10^{31} \mathrm{erg} \mathrm{s}^{-1}$, which allowed us to detect all the qNS's present.

In globular clusters, the number of qNSs is expected to scale with the collision rate which is proportional to $\rho_{0}^{1.5} r_{c}^{2}$ for virialized clusters, where $\rho_{0}$ is

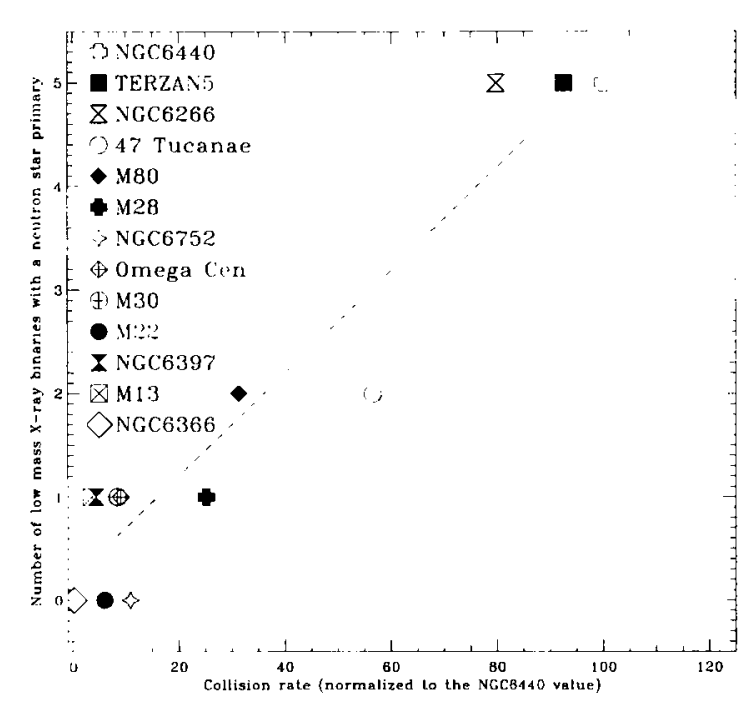

Fig. 1. qNSs versus collision rate (normalized to 100 for NGC 6440) and a linear fit $n_{\mathrm{qNS}} \sim 0.04 \times \rho_{0}^{1.5} r_{c}^{2}+0.2$.

the central density of the cluster and $r_{c}$ its core radius (Verbunt 2002). In Fig. 1, we plot the number of qNSs as a function of the collision rate, normalized so that the value for NGC 6440 is 100 . There is a striking correlation between the number of qNSs and the collision rate. This correlation extends over more than two orders of magnitude and includes both coreand non-core-collapsed clusters. This strongly supports the idea that qNSs are indeed primarily produced by stellar encounters in globular clusters.

\section{REFERENCES}

Brown, E.F., Bildstein, L., \& Rutledge, R.E. 1998, ApJ, 504, L95

Carson, J. E., Cool, A.M., \& Grindlay, J. E. 2000, ApJ, 532,461

Di Stefano, R., \& Rappaport, S. 1994, ApJ, 423, 274

Gendre B., Barret D., \& Webb N. 2003a, A\&A, 400, 521

Gendre B., Barret D., \& Webb N. 2003b, A\&A, 403, L11

Grindlay, J.E., Heinke, C., Edmonds, P.D., \& Murray, S. S. 2001, Sci., 292, 2290

Hasinger G., Altieri B., Arnaud M., et al. 2001, A\&A, $365, \mathrm{~L} 45$

Hertz P., \& Grindlay J. 1983, ApJ, 275, 105

Hut, P., McMillan, S., Goodman, J., et al. 1992, PASP, 104,981

Kaluzny, J., Kubiak, M., Szymanski, M., Udalski, A., Krzeminski, W., \& Mateo, M. 1996, A\&AS, 120, 139

Kirsch M., and the EPIC Consortium 2002, XMM-SOCCAL-TN-0018

Rutledge, R.E., Bildsten, L., Brown, E.F., Pavlov, G.G., \& Zavlin, V. E. 2002, ApJ, 578, 405

Verbunt, F. 2002, ASP Conf. Ser., iNew horizons in globular cluster astronomy, eds. G. Piotto \& G. Meylan

Webb, N.A., Serre, D., Gendre, B., \& Barret, D. 2004, A\&A, submitted 